\title{
Use of Convalescent Plasma for COVID-19 in Pregnancy Lessons from other Viruses
}

\author{
Ahmed M Abbas ${ }^{1,2 *}$, Andro T Fawzy ${ }^{2,3}$, Safaa K Fathy ${ }^{2,4}$, Areej A Abdelazeez ${ }^{2,3}$, Tarek M Essa ${ }^{2,3}$, \\ Omar A Ahmed ${ }^{5}$ and Asmaa S Shaltout ${ }^{6}$ \\ ${ }^{1}$ Department of Obstetrics \& Gynecology, Faculty of Medicine, Assiut University, Egypt.
}

${ }^{2}$ CoViD-19 Research of Assiut University Association (CORAUNA) group, Egypt.

${ }^{3}$ Undergraduate student, Faculty of Medicine, Assiut University, Egypt.

${ }^{4}$ House officer, Faculty of Medicine, Assiut University, Egypt.

${ }^{5}$ Department of Pathology, Faculty of Medicine, Assiut University, Egypt.

${ }^{6}$ Department of Medical Microbiology and Immunology, Faculty of Medicine, Assiut University, Egypt.

*Corresponding author: Ahmed M Abbas, Professor at Department of Obstetrics and Gynecology, Assiut University, Egypt, Women Health Hospital, Assiut, Egypt.

To Cite This Article: Ahmed M Abbas. Use of Convalescent Plasma for COVID-19 in Pregnancy

Lessons from other Viruses. 2020 - 9(6). AJBSR.MS.ID.001443. DOI: 10.34297/AJBSR.2020.09.001443.

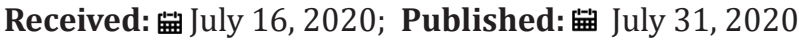

\begin{abstract}
Since the appearance of coronavirus disease-2019 (COVID-19), scientists and physicians work together to find any solution to the current pandemic. Up till now, there is neither definite curative treatment nor vaccine to this infection. Pregnant women are a vulnerable category of population. In normal situation, they need special care. This care must be needed now to protect them and their fetuses. In this review, we highlighted the use of Convalescent Blood Plasma (CBP) in the treatment of pregnant women with COVID-19 infection. It is not new for us to use CBP in the treatment of viral infections as we used before in the treatment of SARS, MERS and other members of coronaviruses family and showed great results in the cure of the patients.
\end{abstract}

Key words: COVID-19, Coronavirus, Convalescent blood plasma, Pregnancy, Pandemic, Wuhan, Pneumonia, Healthy adults, Cough, Fever

\section{Introduction}

In December 2019, many cases of acute pneumonia of unknown cause appeared in Wuhan, China. This pneumonia got its chance to spread throughout China and then has invaded the whole world. After that it was discovered to be caused by a novel virus belongs to the coronaviruses family and the World Health Organization (WHO) named it coronavirus disease-2019 (COVID-19) [1]. This family includes other members like SARS, H5N1, H1N1 and MERS, but COVID-19 got the fastest spread in comparison to them. In normal situations, pregnant females need special care seeking for the best pregnancy outcomes. But during the current situation of COVID-19 pandemic, those females need more care and attention especially after the appearance of adverse outcomes on the mother and her baby by this virus [2].

\section{Effect on mother}

All available evidence suggests that pregnant women are at no greater risk of becoming seriously unwell than other healthy adults if they develop coronavirus. Most pregnant women experience only mild or moderate cold/flu-like symptoms. Cough, fever, shortness 
of breath, headache, and loss of sense of smell are other relevant symptoms [3]. However, mothers still can die from COVID-19 respiratory complications. Two mothers died after delivery due to respiratory complications [4].

\section{Effect on fetus}

Up to date, there is no evidence of intrauterine vertical transmission of COVID-19. Investigations like RT-PCR in cord blood, amniotic fluid or placenta were negative for the virus. However, there are reports of neonatal infection [5]. Those infants' blood samples had antiviral antibodies in their blood after birth [6]. Unfortunately, IgM antibodies are too large to transfer through the placenta to the fetus, and this raises the suggestion of postnatal infection [7]. A study of nine pregnant women who were infected with COVID-19 and had symptoms showed that none of their babies was affected by the virus. The virus was not present in amniotic fluid, the babies' throats, or in breast milk [8]. Another study of 38 women infected with COVID-19 found that none of the newborns tested positive for the disease [9].

To date, all studies showed fetal infection in the third trimester but no available data about the first or second trimester. According to a systematic review of 17 studies, fetal affection appeared in the form of fetal distress, preterm birth, low birth weight, stillbirth neonatal asphyxia, and neonatal death [10]. A simple explanation for that is in China, a lot of cases of pregnancies with suspected or confirmed COVID-19 have been treated and delivered in several hospitals with no maternal death. While the infection occurs in the third trimester of pregnancy, there may be some risks of premature rupture of membranes, this may lead also to premature labor and the baby may suffer from tachycardia and distress [11]. Thus, in turn, we are in a compelling need for finding a treatment for COVID-19 infection to protect those mothers and their babies.

\section{Immunity in Pregnancy}

For a long time, pregnancy was thought of as a state of immunosuppression, but it is now more like a myth, now it is rather a state of immune modulation, which also varies in different phases of pregnancy. During pregnancy there are two immune systems working together, the maternal immune system and the feto-placental which can participate actively in response to infections and modulate maternal response [12]. Also, nature of immunity varies in different phases of pregnancy, first trimester is pro-inflammatory, second trimester is anti-inflammatory, third trimester is pro-inflammatory. So, maternal response can be affected by the type of organism, stage of pregnancy and feto-placental part response to infection.

\section{Response to Viral Infection in Pregnancy}

It looks like that immunological response of placenta can guide maternal immune response. So, if placental infection was associat- ed with severe inflammatory response and release of cytokines as TNF alpha, IFN gamma, IL-6, this will lead to activation of maternal immunity and subsequent miscarriage or preterm labor

If placental infection was associated with mild response that can lead to activation of maternal immune system with no termination of pregnancy and its sensitization to other viruses besides, activation of fetal immune system \& inflammatory response even without viral transmission. This fetal inflammatory response is a condition in which there is high level of circulating cytokines while there are not cultivable organisms [13]. These cytokines were associated with CNS and CVS abnormalities [14].

\section{Active Role of Trophoblast Against Viral Infection}

During viral replication, DsRNA of the virus is produced and recognized by TLR3 in trophoblast leading to:

A. Production of anti-viral factors as Secretory Leukocyte Protease Inhibitor (SLBI) \& IFN beta [15]

B. Production of chemokines such as KC, G-CSF, IL12P40, MCP-1, etc. which will attract the immune cells towards placenta leading to edema, necrosis, hemorrhage in the placenta \& fetal membranes.

C. Production of cytokines as TNF alpha, IFN gamma, IL-6 which regulates prostaglandin production, which is responsible for uterine contraction, these cytokines may also induce apoptosis of trophoblast [16].

\section{Adaptive Immunity in Pregnancy}

As pregnancy is a unique situation with the presence of fetus \& his mother's need to tolerate his existence, modulation of her immunity ensues to secure his existence. Here we give a look at the modulation that happens in adaptive immunity.

\section{T cells}

Pregnancy was noticed to be associated with decrease in T-lymphocytes including CD4 \& CD8 T cells [17] and increase in $\mathrm{T}$ regulatory cells which suppress $\mathrm{T}$ cell proliferation and induce self-tolerance [18]. Focusing on T helper cells, pregnancy is found to be strongly associated with physiological shift to Th2 dominant environment with secretion of Th2 cytokines including IL-4, IL-10 which dampen Th1 type immunity \& this is important for successful pregnancy [19].

\section{B-lymphocytes}

Some studies show that pregnancy is associated with decreased B cell lymphopoiesis, B-lymphocytopenia, decrease in function of B cells \& response to mitogens [20]. However, pregnancy effect on humeral immunity is complicated \& weird. While there is inhibited B cell lymphopoiesis in pregnancy, estrogen administration in vitro was associated with higher IgM \&IgG production from peripheral 
blood mononuclear cells \& in vivo was associated with increase of autoantibodies in SLE mouse models. So, it looks like estrogen is associated with enhanced B lymphocyte action [21]. So, how comes with suppressed lymphopoiesis \& lymphocytopenia noticed in pregnancy. Previous data suggests that pregnancy is associated with inhibition of new B cell development but promoting the already mature $B$ cells with enhanced antibody production of these mature B cells. This can be evidenced by finding no loss of antibody response to inactivated influenza vaccine administration during pregnancy in this study in which the response to the influenza vaccine was rather dependent on memory B cell due to previous exposure to similar virus strains with similar antigens [22].

\section{Changes in Serum Cytokines and other Components in Pregnancy}

Pregnancy in previous studies was found to be associated with significant decrease in GM-CSF, MCP-1, IFN-Y, significant increase in G-CSF \& TNF alfa [23], and significant increase in Alfa defensins 1-3 in blood in pregnancy. Alfa defensins are anti-microbial peptides with defense properties against bacteria, fungi \& enveloped viruses [24].

\section{COVID-19 and Immunity}

\section{The Innate Immunity}

The innate immunity seems the first line of defense against COVID-19, it depends mainly on release of IFN-1which can inhibit viral replication and induce adaptive immune response [24]. On looking at previous corona viruses, SARS \& MERS, they both adopted strategies to suppress IFN-1 [25]. This suppression strategy was associated with disease severity. So, weak initial response, immunity deregulation \& suppressed IFN-1 can interfere with early viral control leading to influx of hyper-inflammatory macrophages with release of high levels of cytokines possibly leading to ARDS \& multi-organ failure [25]. While strong initial response can lead to control of viral replication \&attraction of specific T-cells resulting in elimination of infection with minimal damage to lungs [25].

\section{Adaptive Immunity}

\section{T-lymphocytes Status in COVID-19}

COVID-19 was found to be associated with lymphocytopenia mainly affecting T-lymphocytes, specifically CD4+ cells but no significant change in CD8+/B Cells [24]. This raises the question; why is there lymphocytopenia noticed in COVID-19 patients?

This could mainly attribute to the T-lymphocytes attraction to the lungs due to releases cytokines \& chemokines which can attract monocytes and lymphocytes but not neutrophils. The thing that explains lymphocytopenia \& increased N/L ratio associated with covid19 patients [25]. But interestingly, cytokines elevated with COVID-19 including: IL-1B, IL-2, IL-7, IL-8, IL-9, IL-10, IL-17, GCSF, GM-CSF, IFN Y, TNF alfa, IP10, MCP1, MIP1A, MP1B indicate high response of Th1, Th2, Th17 cells. This may show a conflict with lymphocytopenia. In fact, there is already CD4+ lymphocytopenia because they are attracted to lungs, to combat the infection \& while they are there, they secrete many cytokines, and this extensive response may result in damage of the lung itself.

\section{COVID-19 and the Cytokine Storm}

Cytokine storm simply is excessive immune response to a stimulus resulting in release of many cytokines which can have deleterious effects on body organs including lungs and other organs. It is now known that COVID-19 in some patients is associated with cytokine storm leading to high morbidity \& mortality [26]. Cytokine storm is a major cause of Acute Respiratory Distress Syndrome (ARDS), multi-organ failure [27]. Studies are still carried out to better recognize \&understand the exact mechanisms, but so far, through looking back to SARS, MERS \& also current information about COVID-19, it looks like to be caused by:

A. Suppression of IFN-1 response, loss of early viral control leading to invasion of hyper inflammatory monocytes \& release of many pro-inflammatory cytokines [28]. To better understand this, during viral attack, innate immune response is the first line of defense against this invasion, this innate response depends mainly on IFN-I \& its cascade. Corona viruses including SARS, MERS \& possibly COVID-19 adopt strategies to dampen the initial IFN-1 response leading to loss of early viral control, increased viral replication, high levels of pyroptosis - programmed cell death with highly inflammatory form [25]. Suppression of early IFN-1 response will lead to invasion of hyper inflammatory monocytes with release of many pro-inflammatory cytokines. While increased pyroptosis leads to elevation of IL-1B [29]. Suppressed IFN-I response, increased pyroptosis, detection of Pathogen Associated Molecular Patterns (PAMPs) such as viral RNA by Pattern Recognition Receptors (PRRs) of alveolar macrophages will lead to local inflammation with release of high level of cytokines \& chemokines causing the attraction of monocytes \& T cells from blood to infected region leading to even higher levels of cytokines.

B. Presence of highly inflammatory macrophages in broncho-alveolar lavage fluid \& high CD4+CD16+ inflammatory monocytes in blood -specially in severe COVID-19 patients rather than mild ones - was associated with secretion of inflammatory cytokines as MCP1, MIP1 alfa, IP-10 contributing to the cytokine storm [30].

C. Possible infection of immune cells as monocytes by COVID-19 virus can lead to aberrant cytokine secretion as found in earlier studies of SARS-CoV [31].

\section{Convalescent Blood Plasma}

Convalescent Blood Plasma (CBP) transfusion depends mainly on separating blood plasma from individuals whom have success- 
fully overcome infection, using the specific antibodies-evolved by their immune system- or combat the same invader in other patients, with main concern to eradicate the pathogen [32]. These specific Abs, as well as convalescent plasma an IVIg, has mechanisms of action:

A. Neutralization the Infection, which means that they will Bind to the Virus, prevents its Entry to Cells \& so Deleting its Infectivity, Preventing its Amplification [33].

B. Complement Activation via Classical Pathway, once the Abs Bind to Pathogen via Fab Region, C1 Complexes Bind to Fc Region, starting a Cascade Leading to Formation of Membrane Attack Complex \& So to Lysis of The Virus [34].

C. Antibody Dependent Cellular Toxicity, in which Abs Bind to Virus via Fab \& Bind with Natural Killer Cells Via Fc Region, so in Short Antibodies do the Identification \& Leave the Dirty Work-Killing-for Natural Killer Cells with even Making these Natural Killers more Professional \& Effective.

D. Agglutination, In Which Abs Work as Glue \& Form Clumps of Foreign Bodies Which Are Attractive for Phagocytosis.

E. Also, Interestingly, IgG can pass to Fetus by Placenta, providing the Baby with Temporary Protection.

F. Curiously, this Convalescent Plasma does not only have Anti-Viral Properties, but also it has Immuno-Modulatory Effects which can be helpful in COVID-19 Patients.

\section{Anti-Autoimmune Disorder Properties}

Interestingly, some critically ill COVID-19 patients tested positive for presence of ant phospholipid Abs [35], so the use of CBP may neutralize these Antibodies, reducing possibility of associated complications as coagulopathy \& associated pregnancy complication as miscarriage, early delivery, oligohydramnios, fetal distress, neonatal thrombosis, pre-eclampsia and eclampsia [36].

CBP can solve this problem through inclusion of anti-idiotypic Abs during preparation of IVIg can block auto reactive antibodies of the recipients \& so can be used in autoimmune disorders.

Early clearance of autoimmune Abs by shortening their lifetime by saturating neonatal Fc receptors with IVIg [37]. This neonal Fc Repertory (FcRn) can control the lifetime of IgG by preventing its degradation [38].

\section{Anti-complement properties}

Some Abs can inhibit C3a, C5a which play a significant role in inflammation \& allergic reaction [39].

\section{Anti-cytokine properties}

IgG in plasma can neutralize some cytokines as IL-1B, TNF Alfa [40]

\section{Convalescent Plasma Immuno-Modulators Effect on T-Cells}

Using IVIg was associated with decrease of Th1 cells, low IFN Y, TNF Alfa, decreases proliferation of Th17 cells, decreased IL-17, elevated Th2 cytokines [41], reduction \& decreased activation of cytotoxic T cells, improved pregnancy outcome in cases with recurrent pregnancy loss due to its immunomodulation properties of Th1, Th2, and Th17/Treg ratio [42].

\section{CBP Immunomodulatory Effects on Innate Immunity Cells including Dendritic Cells \& Macrophages}

\section{Dendritic Cells}

IVIg was found to be associated with promoting anti-inflammatory profile of Dendritic Cells (DCs) via inhibiting maturation of DCs, decreased production of IL-17 increased IL-10 production, IL4, IL-13, and down regulation of co-stimulation molecules (CD40, CD80, CD86), down regulation of HLA-II in DCs [43].

\section{Macrophages}

Treating macrophages with IVIg is associated with enhancement of anti-inflammatory properties of macrophage via decreasing production of IL-12/23p [40] and increased production of IL-10 [44]. While there is no clue indicates that IVIg is associated with inhibiting macrophage migration to lungs, but there was decreased nerve macrophage infiltration in a study made in induced peripheral neurotoxicity in rats [45].

\section{Role of Convalescent Plasma for COVID-19 in Pregnancy}

Theoretically, CBP use in pregnancy is useful in too many ways $\&$ further studies need to be performed to test its efficacy in real world on pregnant women. When it comes to adaptive immunity, it looks dampened in pregnancy, so using CBP in pregnancy with COVID-19 will provide the pregnant with specific abs against the virus helping her immune system to eradicate the pathogen through anti-viral mechanisms shown before. It also neutralizes the virus, so deleting its interaction with placenta \& trophoblast (via TLR3 as shown before) which can trigger preterm delivery as shown with some COVID-19 pregnant women. Additionally, as IgG can cross the placenta, it will possibly provide the fetus with passive immunity. Finally, through its immune-modulatory \& anti-inflammatory effects, it seems that CBP has properties which can suppress cytokine storm which is a major cause of ARDS \& multi-organ failure.

History of Convalescent Plasma use with other Viral Infections

The concept of immunotherapy takes us back to late $19^{\text {th }}$ century when this concept derived from works of Ehrlich, Pasteur \& Bordeau whom recognized the neutralizing action of antibodies [46]. 
In 1880, it was found that immunity developed in animals-after intentional immunization with nonlethal doses of diphtheria toxins \& which was mainly derived on abs against toxins in the blood of animals-can be transferred to animals with active infections [47]. Then the immune sera of some animals as goats, horses \& sheep were used in humans suffering from diphtheria, it was successful \& saved many lives [48].

In the 50 s of last century, it was available to purify \& concentrate immunoglobulins derived from immunized donors whether by full recovery from past clinical infection or asymptomatic infection \& so formed the hyper immune globulin. Since then, IVIg progressively replaces animal sera use \& it was used in serious infection and immune disorders as immunodeficiency and autoimmune diseases, as immune plasma was found to have immuno-modulatory characteristics besides its ability to neutralize pathogens [49].

Although CBP products as polyclonal abs, monoclonal abs, IVIg have several benefits and used but their use in cases of emergency is limited as their production is difficult \& costly, so in cases of emergency with no other available medication or vaccine, CBP is the first therapeutic option.

\section{Use of CBP in Spanish Flu}

A meta-analysis was performed on the effect of convalescent blood products on patients with Spanish flu, its efficacy, adverse events [50]. It was found that:

A. Use of convalescent plasma products were associated with improved outcome in the form of improved clinical symptoms \& signs, decreased risk of death, decreased mortality with overall crude case fatality rate $16 \%$ in intervention patients versus $37 \%$ in controls.

B. Early treatment with CBP (after $<4$ days of pneumonia complications) was associated with better outcome than late treatment.

\section{Use of CBP in Avian Flu H5N1}

Zhou et al [51] found in a previously healthy male tested positive for H5N1, started treatment with oseltamivir but with no improvement \& the viral load continued to increase, that CBP from another patient who had recovered from H5N1 infection was obtained, resulted in decreased viral load \& even became undetectable within 32 hours after first transfusion. Additionally, there were improved radiographic findings of pulmonary lesions.

\section{Use of CBP in H1N1}

Hung et al [52] in H1N1 patients reported that administration of CBP (with neutralizing antibody titer $>\backslash=1: 160$ ) was associated with significant decrease in the mortality among treatment group vs. non-treatment group ( $20 \%$ vs. $54.8 \%$ ). Another study showed administration of hyper immune IVIg was associated with significantly lower viral load and reduced mortality in H1N1 patients, and no adverse events were reported [53].

\section{Use of CBP in SARS}

Soo et al. [54] reported that CBP administration was associated with decreased length of stay in hospital, decreased mortality (death rate in plasma group was $0 \%$ ) vs. the steroid group who had longer stay \& higher mortality. Cheng et al. [55] found that CBP administration was associated with decreased mortality in general $(12.5 \%$ mortality rate in intervention group vs. $17 \%$ overall SARS related mortality in Hong Kong). Yhe et al. [56] showed an association between CBP administration with decreased viral load, increase in anti SARS-CoV IgM, IgG over time, but the interesting about this study that one patient became pregnant, delivered 13 months after discharge \& the baby was positive for anti SARS Abs suggesting possible transfer of abs from his mother.

\section{Use of CBP in MERS}

Ko et al. [57] reported two of the three patients with MERS treated by CBP showed neutralizing activity \& it was concluded that CBP should be with neutralizing activity of PRNT (Plaque Reduction Neutralization Test) titer $>\backslash=1: 80$.

\section{Lessons from Previous Viruses}

All previous studies had several limitations \& were all exposed to moderate/ high bias chances because of

A. Absence of randomized clinical trials,

B. Absence of intervention and control groups in most of previous studies,

C. The dose, timing, antibody neutrality titer, CBP volumes were not standardized,

D. Use of CBP was not alone \& there were adjunctive therapy next to it, so its isolated efficacy cannot be determined,

E. Reports about its use in pregnancy are rare.

However, there are some lessons that can be learnt from previous data; it is noticed that early use of CBP is associated with better outcome. this can be explained by most viruses reaching their peak viremia in the first week of infection followed by development of primary response between days 10-14, so CBP administration should be early in the course of viral infection and in correspondence with peak viremia. Also, neutralizing Ab titer is important thing to consider as seen in Ko et al. study [57] about MERS where antibody neutralizing titer should be $\geq 1: 80$ to achieve neutralizing activity \& to avoid antibody dependent enhancement with sub-neutralizing concentrations. Therefore, plasma should be collected from recently recovered patients as humoral immune response is short lasting \& Ab titer decreases rapidly [58]. Increase the load on 
circulation is a severe complication of CBP transfusion. Also, RBCs alloimmunization, hemolytic transfusion reactions and febrile non hemolytic transfusion reactions are rare and not considered sever complications. Many theories consider mortality rates in patients with any disease other than trauma or diseases related to trauma increased. Also, morbidity and mortality increased in case of compatible but not identical ABO plasma and mortality tendency increases much more after transfusion by about 14 days. In contrast, many theories consider that plasma transfusion is not associated with mortality and never cause it, but it surely has its severe complications.

\section{Conclusion}

In conclusion, use of CBP in pregnant women should be considered; it forms a potential effective therapy for COVID-19 in pregnant patients and appears to be safe. Through history of use, it has a significant effect at improving clinical outcome \& decreasing mortality rate. Through lessons from CBP use, the dose and neutralizing $\mathrm{Ab}$ titer should be considered, as sub-neutralizing may lead to antibody dependent enhancement phenomenon. Transfusion rate $\&$ dose should be adjusted in pregnant women to avoid transfusion associated circulatory overload as pregnancy is associated with cardiovascular system changes which includes increased whole blood volume \& cardiac output. Timing of CBP administration is important, and early timing in correspondence with peak viremia was significantly associated with better outcome than late timing, so use of CBP before day 14 of the disease should be considered. Adverse events with CBP use were rare in general \& mostly mild, so CBP use looks safe.

\section{Reference}

1. Park SE (2020) Epidemiology, virology, and clinical features of severe acute respiratory syndrome-coronavirus-2 (SARS-CoV-2; Coronavirus Disease-19). Clin Exp Pediatri 63(4): 119-24.

2. Zhao X, Jiang Y, Zhao Y, Xi H, Liu C, et al. (2020) Analysis of the susceptibility to COVID-19 in pregnancy and recommendations on potential drug screening. Eur J Clinl Microbiol Infect Dis 23: 1-12.

3. Khan S, Peng L, Siddique R, Nabi G, Xue M, et al. (2020) Impact of COVID-19 infection on pregnancy outcomes and the risk of maternalto-neonatal intrapartum transmission of COVID-19 during natural birth. Infect Control Hosp Epidemiol 19: 1-3.

4. Karimi ZM, Neamatzadeh H, Dastgheib SA, Abbasi H, Mirjalili SR, et al. (2020) Vertical transmission of coronavirus disease 19 (COVID-19) from infected pregnant mothers to neonates: a review. Fetal Pediatr Pathol $1: 1-5$.

5. Li M, Xu M, Zhan W, Han T, Zhang G, et al. (2020) Report of the first cases of mother and infant infections with 2019 novel coronavirus in Xinyang City Henan Province. Chin J Infect Dis 12: 7.

6. Dong L, Tian J, He S, Zhu C, Wang J, et al. (2020) Possible vertical transmission of SARS-CoV-2 from an infected mother to her newborn. JAMA 323(18): 1846-1848.

7. Chen S, Huang B, Luo DJ, Li X, Yang F, et al. (2020) Pregnant with new coronavirus infection: a clinical characteristics and placental pathological analysis of three cases. Chin J Pathol 49(5): 418-423.
8. Yan J, Guo J, Fan C, Juan J, Yu X, et al. (2020) Coronavirus disease 2019 (COVID-19) in pregnant women: A report based on 116 cases. Am J Obstet Gynecol 223(1): 111.1-111.14.

9. Schwartz DA (2020) An analysis of 38 pregnant women with COVID-19, their newborn infants, and maternal-fetal transmission of SARS-CoV-2: maternal coronavirus infections and pregnancy outcomes. Arch Pathol Lab Med 55(6): 841-843.

10. Yang Z, Wang M, Zhu Z, Liu Y (2020) Coronavirus disease 2019 (COVID-19) and pregnancy: a systematic review. J Maternal Fetal Neonat Med 30: 1-4.

11. Liang H, Acharya G (2020) Novel corona virus disease (COVID-19) in pregnancy: What clinical recommendations to follow? Acta Obstet Gynecol Scand 99(4): 439-442.

12. Mor G, Cardenas I (2010) The immune system in pregnancy: a unique complexity. Am J Reprod Immunol 63(6): 425-433.

13. Romero R, Gotsch F, Pineles B, Kusanovic JP (2007) Inflammation in pregnancy: its roles in reproductive physiology, obstetrical complications, and fetal injury. Nutr Rev 65(suppl_3): 194-202.

14. Deverman BE, Patterson PH (2009) Cytokines and CNS development. Neuron 64(1): 61-78.

15. Abrahams VM, Schaefer TM, Fahey JV, Visintin I, Wright JA, et al. (2006) Expression and secretion of antiviral factors by trophoblast cells following stimulation by the TLR-3 agonist, Poly (I: C). Human Reprod 21(9): 2432-2439.

16. Hansen WR, Keelan JA, Skinner SJ, Mitchell MD (1999) Key enzymes of prostaglandin biosynthesis and metabolism. Coordinate regulation of expression by cytokines in gestational tissues: a review. Prostag Oth Lipid M 57(4): 243-257.

17. Kraus TA, Engel SM, Sperling RS, Kellerman L, Lo Y, et al. (2012) Characterizing the pregnancy immune phenotype: results of the viral immunity and pregnancy (VIP) study. J Clin Immunol 32(2): 300-311.

18. Kondelkova K, Vokurková D, Krejsek J, Borská L, Fiala Z, et al. (2010) Regulatory T cells (TREG) and their roles in immune system with respect to immunopathological disorders. Acta Medica 53(2): 73-77.

19. Raghupathy R (1997) Th 1-type immunity is incompatible with successful pregnancy. Immunol Today 18(10): 478-482.

20. Lima J, Martins C, Leandro MJ, Nunes G, Sousa MJ, et al. (2016) Characterization of $\mathrm{B}$ cells in healthy pregnant women from late pregnancy to post-partum: a prospective observational study. BMC Pregnancy Childbirth 16(1): 139.

21. Grimaldi CM, Michael DJ, Diamond B (2001) Cutting edge: expansion and activation of a population of autoreactive marginal zone B cells in a model of estrogen-induced lupus. J Immunol 167(4): 1886-1890.

22. Kraus TA, Sperling RS, Engel SM, Lo Y, Kellerman L, et al. (2010) Peripheral blood cytokine profiling during pregnancy and post-partum periods. Am J Reprod Immunol 64(6): 411-426.

23. Klotman ME, Chang TL (2006) Defensins in innate antiviral immunity. Nature Rev Immunol 6(6): 447-456.

24. Qin C, Zhou L, Hu Z, Zhang S, Yang S, et al. (2020) Dysregulation of immune response in patients with COVID-19 in Wuhan, China. Clinical Infectious Diseases 248.

25. Tay MZ, Poh CM, Rénia L, MacAry PA, Ng LF (2020) The trinity of COVID-19: immunity, inflammation and intervention. Nature Rev Immunol 28: 1-2.

26. Mehta P, McAuley DF, Brown M, Sanchez E, Tattersall RS, et al. (2020) COVID-19: consider cytokine storm syndromes and immunosuppression. Lancet 395(10229): 1033-1034. 
27. Chousterman BG, Swirski FK, Weber GF (2017) Cytokine storm and sepsis disease pathogenesis. In Seminars in immunopathology 39(5): 517-528.

28. Prompetchara E, Ketloy C, Palaga T (2020) Immune responses in COVID-19 and potential vaccines: Lessons learned from SARS and MERS epidemic. Asian Pac J Allergy Immunol 38(1): 1-9.

29. Huang C, Wang Y, Li X, Ren L, Zhao J, et al. (2020) Clinical features of patients infected with 2019 novel coronavirus in Wuhan, China. Lancet 395(10223): 497-506.

30. Liao M, Liu Y, Yuan J, Wen Y, Xu G, et al. (2020) The landscape of lung bronchoalveolar immune cells in COVID-19 revealed by single-cell RNA sequencing. MedRxiv.

31. Cheung CY, Poon LL, Ng IH, Luk W, Sia SF, et al. (2005) Cytokine responses in severe acute respiratory syndrome coronavirus-infected macrophages in vitro: possible relevance to pathogenesis. J Virol 79(12): 7819-26.

32. Burnouf T, Seghatchian J (2014) Ebola virus convalescent blood products: where we are now and where we may need to go. Transfus Apher Sci 51(2): 120-125.

33. Du L, He Y, Zhou Y, Liu S, Zheng BJ, et al. (2009) The spike protein of SARS-CoV-a target for vaccine and therapeutic development. Nature Rev Microbiol 7(3): 226-236.

34. Wu D, Yang XO (2020) TH17 responses in cytokine storm of COVID-19: An emerging target of JAK2 inhibitor Fedratinib. J Microbiol Immunol Infect 53(3): 368-370.

35. Zhang Y, Xiao M, Zhang S, Xia P, Cao W, et al. (2020) Coagulopathy and antiphospholipid antibodies in patients with Covid-19. New Eng J Med 382(17): 38.

36. Di Prima FA, Valenti O, Hyseni E, Giorgio E, Faraci M, et al. (2011) Antiphospholipid Syndrome during pregnancy: the state of the art. J Prenatal Med 5(2): 41-53.

37. Rossi F, Jayne DR, Lockwood CM, Kazatchkine MD (1991) Anti-idiotypes against anti-neutrophil cytoplasmic antigen autoantibodies in normal human polyspecific IgG for therapeutic use and in the remission sera of patients with systemic vasculitis. Clin Exp Immunol 83(2): 298-303.

38. Mouthon L, Kaveri SV, Spalter SH, Lacroix DS, Lefranc C, et al. (1996) Mechanisms of action of intravenous immune globulin in immunemediated diseases. Clin Exp Immunol 104: 3-9.

39. Hugli TE, Müller Eberhard HJ (1978) Anaphylatoxins: C3a and C5a. Inadvances in immunology. Academic Press 26: 1-56.

40. Abe Y, Horiuchi A, Miyake M, Kimura S (1994) Anti-cytokine nature of natural human immunoglobulin: one possible mechanism of the clinical effect of intravenous immunoglobulin therapy. Immunol Rev 139(1): 5-19.

41. Ahmadi M, Vahid AS, Ghaebi M, Maleki AL, Afkham A, et al. (2017) Effect of intravenous immunoglobulin on Th1 and Th2 lymphocytes and improvement of pregnancy outcome in recurrent pregnancy loss (RPL). Biomed Pharmacother 92: 1095-1102.

42. Kim DJ, Lee SK, Kim JY, Na BJ, Hur SE, et al. (2014) Intravenous Immunoglobulin G Modulates Peripheral Blood Th17 and F oxp3+ Regulatory T Cells in Pregnant Women with Recurrent Pregnancy Loss. Am J Reprod Immunol 71(5): 441-450.
43. Bayry J, Desmazes LS, Carbonneil C, Misra N, Donkova V, et al. (2003) Inhibition of maturation and function of dendritic cells by intravenous immunoglobulin. Blood 101(2): 758-765.

44. Kozicky LK, Zhao ZY, Menzies SC, Fidanza M, Reid GS, et al. (2015) Intravenous immunoglobulin skews macrophages to an antiinflammatory, IL-10-producing activation state. J leukocyte Biol 98(6): 983-994.

45. Meregalli C, Marjanovic I, Scali C, Monza L, Spinoni N, et al. (2018) High-dose intravenous immunoglobulins reduce nerve macrophage infiltration and the severity of bortezomib-induced peripheral neurotoxicity in rats. J Neuroinflammation 15(1): 232

46. Finegold I, Dockhorn RJ, Ein D, Dolen WK, Oppenheimer J, et al. (2010) Immunotherapy throughout the decades: from Noon to now. Ann Allergy Asthma Immunol 105(5): 328-336.

47. Shahani L, Singh S, Khardori NM (2012) Immunotherapy in clinical medicine: historical perspective and current status. Med Clin N Am 96(3): 421-431.

48. Graham BS, Ambrosino DM (2015) History of passive antibody administration for prevention and treatment of infectious diseases. Curr Opin HIV AIDS 10(3): 129-134.

49. Garraud O, Heshmati F, Pozzetto B, Lefrere F, Girot R, et al. (2016) Plasma therapy against infectious pathogens, as of yesterday, today and tomorrow. Transfusion Clinique et Biologique 23(1): 39-44.

50. Luke TC, Kilbane EM, Jackson JL, Hoffman SL (2006) Meta-analysis: convalescent blood products for Spanish influenza pneumonia: a future H5N1 treatment? Ann Intern Med 145(8): 599-609.

51. Zhou B, Zhong N, Guan Y (2007) Treatment with convalescent plasma for influenza A (H5N1) infection. New Eng J Med 357(14): 1450-1451.

52. Hung IF, To KK, Lee CK, Lee KL, Chan K, et al. (2011) Convalescent plasma treatment reduced mortality in patients with severe pandemic influenza A (H1N1) 2009 virus infection. Clin Infect Dis 52(4): 447-456.

53. Hung IF, To KK, Lee CK, Lee KL, Yan WW, et al. (2013) Hyperimmune IV immunoglobulin treatment: a multicenter double-blind randomized controlled trial for patients with severe 2009 influenza A (H1N1) infection. Chest 144(2): 464-473.

54. Soo YO, Cheng Y, Wong R, Hui DS, Lee CK, et al. (2004) Retrospective comparison of convalescent plasma with continuing high-dose methylprednisolone treatment in SARS patients. Clinical Microbiol Infect 10(7): 676-678.

55. Cheng Y, Wong R, Soo YO, Wong WS, Lee CK, et al. (2005) Use of convalescent plasma therapy in SARS patients in Hong Kong. Eur J Clin Microbiol Infect Dis 24(1): 44-46.

56. Yeh KM, Chiueh TS, Siu LK, Lin JC, Chan PK, et al. (2005) Experience of using convalescent plasma for severe acute respiratory syndrome among healthcare workers in a Taiwan hospital. J Antimicrob Chemoth 56(5): 919-922.

57. Ko JH, Seok H, Cho SY, Ha YE, Baek JY, et al. (2018) Challenges of convalescent plasma infusion therapy in Middle East respiratory coronavirus infection: a single centre experience. Antivir Ther 23(7): 617-622.

58. Duan K, Liu B, Li C, Zhang H, Yu T, et al. (2020) Effectiveness of convalescent plasma therapy in severe COVID-19 patients. Proc Natl Acad Sci 117(17): 9490-9496. 